\title{
DECISION SUPPORT FOR FIELD QUALITY PROBLEMS UNDER UNCERTAINTY
}

\author{
Minoru Nakatsugawa
}

\author{
Takeichiro Nishikawa \\ Toshiba Corporation
}

Ryusei Shingaki

(Received August 9, 2010; Revised May 16, 2011)

\begin{abstract}
A quick decision on implementation of countermeasures for quality problems is important for reducing the quality cost of electronic products. At the beginning of the production period, the claim rate is uncertain owing to the lack of sufficient field claim data. Therefore, a quick decision on implementation of countermeasures may lead to misjudgment. On the other hand, until the end of the production period, there is an option to postpone the decision. When the option to postpone the decision is exercised, increased field claim data will reduce the uncertainty of the claim rate. Then the probability of misjudgment will decrease. However, postponing the decision may increase the number of inferior products before the countermeasures. In order to support the decision on implementation of countermeasures for quality problems under uncertainty, we propose a new method that combines Bayesian updating and real options. We evaluate the value of the option to postpone the decision qualitatively and show the possibility of reducing the quality cost.
\end{abstract}

Keywords: Decision making, OR practice, Markov decision process, reliability, risk management, real option, optimal stopping, dynamic programming, Bayesian statistics

\section{Introduction}

Quality problems of consumer electronics are critical both for customers and manufacturers. For customers, quality problems decrease the sense of reassurance. For manufacturers, the increase of the quality cost has a huge impact on profitability. The warranty accruals of manufacturers usually account for some percentages of product sales [23]. In addition, the brand image of products is easily damaged by quality problems.

Manufacturers have been devoting much effort to the improvement of product quality in the design process and the production process. However, quality problems are not completely eliminated before shipment. In the case of consumer electronics, field quality problems sometimes become apparent during the production period. In order to improve the product quality of the remaining production period, countermeasures for the quality problems must be implemented before the end of the production period. Since recent electronic products, such as computers, are produced within a short period, a quick decision on implementation of countermeasures is required. Manufacturers must decide quickly whether to implement countermeasures and bear the associated cost.

At the beginning of the production period, uncertainty of claim rates makes the decision difficult owing to the lack of sufficient field claim data. Figure 1 (below) shows a typical change of the amount of in-warranty products. When a new product model begins to be shipped, in-warranty products are few. The amount of in-warranty products increases as the production continues. Then it remains constant, because the production period is usually shorter than the warranty period, which is typically one year. Finally, it begins to decrease one year after the start of production. 


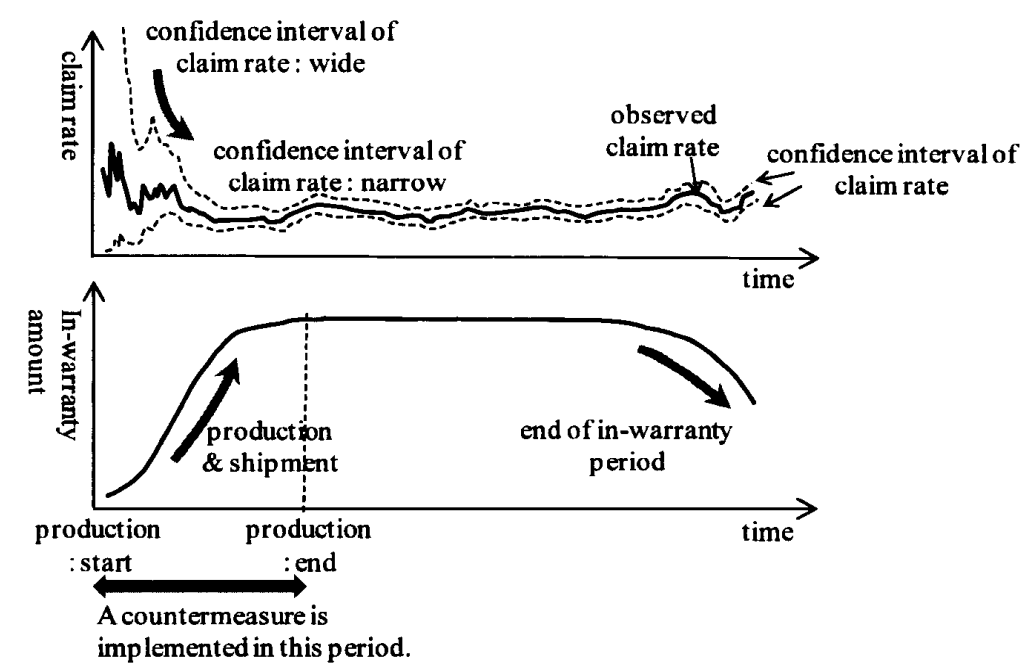

Figure 1: Observed claim rate and two-sided 95\% confidence interval of the claim rate (above), in-warranty amount of products (below)

Figure 1 (above) shows the observed claim rate (solid line) and the two-sided $95 \%$ confidence interval of the claim rate (dotted line). Here, the observed claim rate is calculated by dividing the number of claims by the number of in-warranty products. The confidence interval is calculated based on binomial distribution and considered as uncertainty in decision making. The confidence interval is wide at the beginning of the production period because there are few observed claims and small in-warranty amounts. In other words, the claim rate is uncertain at the beginning of the production period. If the true claim rate is high, an earlier countermeasure has a greater cost-reduction effect. However, the true claim rate may be low. In this case, the countermeasure is ineffective and the expended cost is wasted. Therefore, although manufacturers want to implement countermeasures earlier, quick decision making involves high risk. In particular, it is especially difficult to judge at the beginning of the production period because of the high uncertainty.

However, it is unnecessary to make a decision under high uncertainty. The decision can be postponed until sufficient field claim data and in-warranty product data are collected. If the decision is postponed, uncertainty decreases and the confidence intervals of claim rates become narrow. This makes it possible to make a more correct decision. This option is called the option to postpone or the option to wait in the terminology of real option analysis $[8,16]$. If this option is exercised, the probability of a wrong decision becomes small. However, the amount of the production after the decision will decrease and the effect of the countermeasure will become smaller. Therefore, there is a trade-off between the probability of misjudgment and the effect of countermeasures. In this uncertain situation, it is helpful to support the decision making on whether the manufacturer should implement a countermeasure at the present time or postpone the decision.

Real option analysis is a method of decision making under uncertainty $[8,16]$, developed as an extension of option pricing techniques $[3,15,20]$. Applications of real options have been studied in various domains, such as project investments $[13,18]$, resource investments [4], maintenance planning [9], and policy evaluation [19]. In many industrial projects, management has the option to acquire additional information in order to reduce uncertainty before committing to an irreversible decision [1]. This option is valuable, because the new information reveals more about the true state and reduces risk. From a statistical view- 
point, Bayesian analysis is a natural way to update distributions based on new information. Simple case studies of Bayesian updating are presented in [14] where the reserves are divided into two or three broad categories (high, medium, low) and where the probabilities for each category are conditional upon the true reservoir volume. Once the prior probabilities for each reserve category are known, Bayes' theorem can be used to deduce the probability of high/medium/low reserves given new observations. Decamps et al. [6] and Klein [12] solved for the optimal investment timing when a firm is uncertain about the drift parameter of the state process and the variance parameter is known. In their models, over time the firm learns more about the true drift parameter. In the fullness of time, full information can be obtained. They applied arithmetic Brownian motion to the value of the project, which is thought to be unrealistic.

Optimal stopping is concerned with the problem of choosing a time to take a particular action in order to maximize an expected reward or minimize an expected cost. There are many researches related to the optimal stopping problem. In the area of real option, optimal stopping is related to the pricing of American options [10,11,22]. We consider a kind of an optimal stopping problem for the decision making of implementing a countermeasure against field quality problems at present time, where the number of claims follows binomial distribution. Our criterion function is the expected loss caused by postponing the decision. In Markov decision processes many authors propose a variety of criteria (e.g., utility, probabilistic constraints and mean-variance) and investigate Markov decision processes for their criteria, instead of standard criteria, that is, the expected discounted total reward and the average expected reward per unit [17]. White [24] reviews the decision problems with such criteria in detail. Our problem is related to a parking problem, because our criterion function depends on the stopping time when a countermaeasure (i.e., parking) is implemented. A criterion in a classical parking problem is the expectation reward which is maximized with respect to stopping time. For this problem, for example, see Chow et al. [5] and DeGroot [7]. However, we are not interested in when to take an action unlike general optimal stopping problems, though our proposed method solves a kind of an optimal stopping problem for each simulation scenario. Our simulation scenarios differ by the unobserved true claim rate and the optimal time of implementing a countermeasure or the optimal decision at present time also differs. We repeat the simulation with different true claim rate and calculate the expected loss caused by postponing the decision at the present time for decison making.

Our aim in this paper is to apply a more general approach of Bayesian updating to the decision making for a countermeasure for field quality problems. Firstly, we treat obeserving the amount of in-warranty products and the amount of claims in Bayesian updating framework. The new information can change the estimates of the claim rate. The value of postponing the judgment for implementing a countermeasure to a future decision point is presented. Secondly, we consider an appropriate claim generation model based on binomial distribution and the unobserved true claim rate. Contrastingly, many results of real option analysis assume without any reason that the stochastic process is an arithmetic Brownian process or a Wiener process. Thirdly, we consider appropriate future scenarios based on the observed claim data and the claim generation model. Real option analysis requires future scenarios* and leads to the solution that fits the scenarios. Therefore, future scenarios must be considered appropriately.

We propose a new method to support decision making on countermeasures for quality problems under uncertainty. The proposed method is based on decision tree analysis and

${ }^{*}$ For example, increase and decrease of demand, trend of economy, change of price, and so on. 


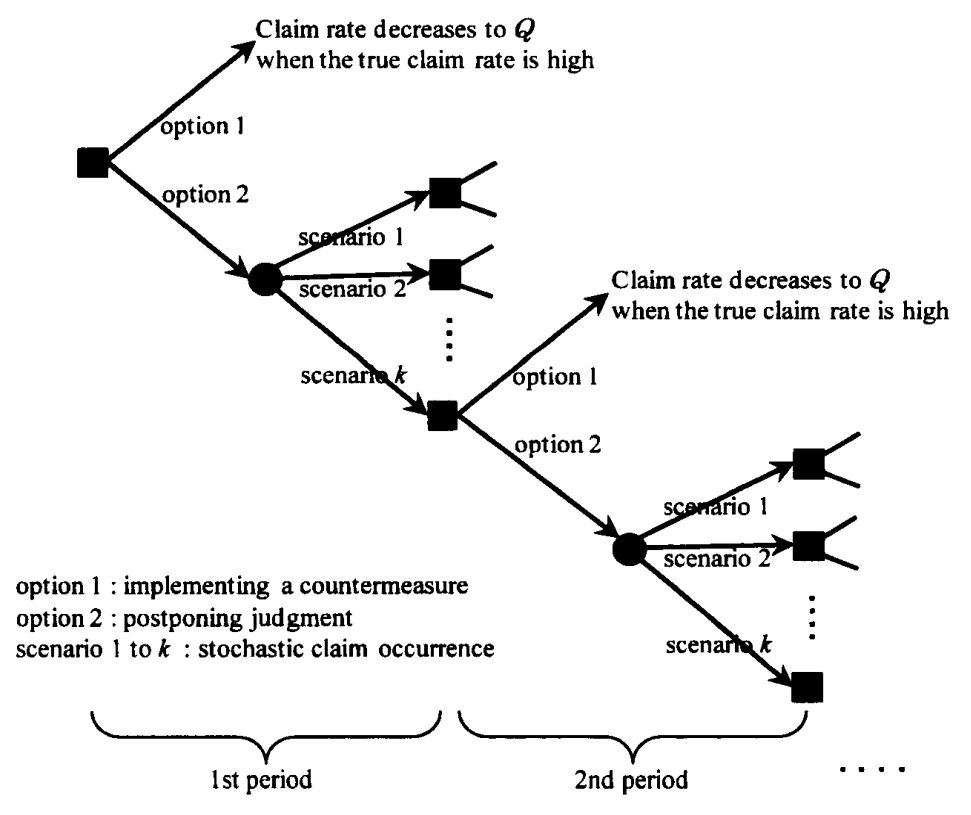

Figure 2: Decision tree

selects the best strategy that minimizes quality cost at each decision point. To the best of our knowledge, there is no study that implements similar methods for the support of decision making for quality problems after shipment.

The remainder of this paper is organized as follows. In Section 2, we introduce the proposed decision-making model and assumptions for the model. The algorithm of our decision-making model is presented in Section 3. Experimental results are shown in Section 4. In Section 5, we conclude the paper.

\section{Decision-making Model and Assumptions}

\subsection{Decision tree}

A decision tree is a rooted tree that represents all possible decisions (options) and scenarios in each period hierarchically. Using a decision tree to find the optimal decisions is called solving a decision tree. Scenarios of a decision tree represent the uncertainty in the future. In the case of implementing countermeasures for quality problems, we deal with the claim rate as the main element of uncertainty.

Figure 2 is a two-period decision tree that illustrates the options and the scenarios of field quality problems. As described in Section 1, we define the following two options at each decision point: implementing a countermeasure and postponing judgment. Implementing a countermeasure means improving the product quality of the remaining production period. Countermeasures include detection of problems, modification of operations, replacement of parts, for example. Improvement of product quality is achieved when the countermeasure is effective. Postponing judgment at time $t$ means implementing no countermeasure at time $t$, but does not mean implementing no countermeasures permanently. Countermeasures can be implemented at any future decision points of the remaining production period after $t$.

Black squares in Figure 2 are decision points. The left-most black square means the decision point at the present time $t_{0}$, and this decision is of interest. At each decision point, the better option is selected by considering whether a countermeasure for quality problems should be implemented now or the judgment should be postponed to the next decision 
point. A decision is made quantitatively by comparing the estimated quality cost of both cases. Here, quality cost means the whole repair cost of the products manufactured after the present time $\left(t_{0}\right)$ and the cost of implementing a countermeasure $(C)$. The cost of a countermeasure includes labor cost and material cost for changing the product design and the production process, for example. Arcs from decision points are called decision arcs, and other arcs from black circles are called scenario arcs. There are two arcs from each of the decision points, which mean implementing a countermeasure and postponing judgment, respectively. Scenario arcs mean stochastic variation of claim amounts in the future. For simplicity of illustration, only three scenario arcs are drawn in Figure 2.

A brief overview of our decision-making model is as follows. The in-warranty products are considered as risk exposure in insurance terminology. Because the claim rate is constant and independent of the elapsed usage time (Assumption 1), it is appropriate to consider that the number of claims $R_{t}$ follows binomial distribution $\operatorname{Bi}\left(N_{t}, p^{*}\right)$.

$$
R_{t} \sim \operatorname{Bi}\left(N_{t}, p^{*}\right)
$$

where $N_{t}$ is a number of in-warranty products and $p^{*}$ is the unobservable true claim rate. This means that claims occur randomly with a constant probability. Following the claim generation model $\mathrm{Bi}\left(N_{t}, p^{*}\right)$, we calculate the realization probability of each $R_{t}$. The amount of $R_{t}$ and $N_{t}$ corresponds to the scenarios of a decision tree at time $t$. Although the claim rate is continuous, we segmented the claim rate into $k$ discrete small grids between 0 and 1 . Scenario arcs are considered discretely as shown in Figure 2. Scenario 1 to $k$ corresponds to the $k$ grids that segment the claim rate between 0 and 1 .

The information that manufacturers get at time $t$ is the number of claims $R_{t}$ and the number of in-warranty products $N_{t}$. The option to postpone is valuable, because the new information $\left(R_{t+1}\right.$ and $\left.N_{t+1}\right)$ reveals more about the true state $\left(p^{*}\right)$. As discussed below, we model this reduction of uncertainty using Bayesian updating.

Based on the decision tree analysis, we calculate the quality cost of each scenario by considering the value of the option to postpone. Then we select the option that minimizes the cost at each decision point. The quality cost at present depends on the decision at the future time. We calculate the quality cost at the future decision point preliminarily in order to calculate the cost at present. To realize this calculation, we use the recursive structure of the problem like dynamic programming.

\subsection{Notations and assumptions}

The notations are listed in Table 1. Before we illustrate the detail of the proposed algorithm, the following assumptions of the presented decision-making model are explained.

1. The claim rate $p^{*}$ is constant and independent of the elapsed usage time.

This means that there are few initial failures and wear-out failures compared with random failures. In general, this is true of electronic devices and systems that consist of many parts [2].

2. If a countermeasure is effective, the claim rate of products manufactured after the countermeasure becomes a fixed value $Q$ : the target claim rate anticipated in the design process. If the true claim rate $p^{*}$ is higher than the target claim rate $Q$, the countermeasure is effective.

We estimate the value of taking a countermeasure and compare it with the quality cost for decision making. In order to estimate the value of taking a countermeasure, we assume that the countermeasure makes the claim rate of products decrease to a fixed rate anticipated in the design process. 
Table 1: List of notations

\begin{tabular}{cl}
\hline Notation & Explanation \\
\hline$t / t_{0} / T$ & time / the present time / time of the final production \\
\hline$p^{*} / q_{i}^{*}$ & true claim rate (unobservable) / true claim rate for $i$ th simulation (unobservable) \\
\hline$g\left(p^{*}\right)$ & posterior distribution of the true claim rate \\
\hline$\hat{p}_{t}$ & observed claim rate at $t: \hat{p}_{t}=R_{t} / N_{t}$ \\
\hline$Q$ & claim rate after implementing a countermeasure \\
\hline$n_{t}$ & amount of in-warranty products at $t$ \\
\hline$N_{t}$ & cumulative sum of in-warranty products at $t: N_{t}=\sum_{\tau=1}^{t} n_{\tau}$ \\
\hline$r_{t}$ & amount of claims at $t$ \\
\hline$R_{t}$ & cumulative sum of claims at $t: R_{t}=\sum_{\tau=1}^{t} r_{\tau}$ \\
\hline$w$ & repair cost of one claim \\
\hline$C$ & cost of implementing a countermeasure \\
\hline$M$ & number of simulations \\
\hline$L_{t}^{A c t}\left(\hat{p}_{t}\right)$ & quality cost when a countermeasure is implemented at $t\left(t_{0} \leq t \leq T\right)$ \\
\hline$L_{t}\left(\hat{p}_{t}, q_{i}^{*}\right)$ & quality cost based on the decision at $t\left(t_{0} \leq t \leq T\right)$ \\
\hline$F_{E}$ & expected loss caused by postponing the decision to $t_{0}+1$ \\
\hline
\end{tabular}

3. Until the end of the production period, it is possible to implement a countermeasure for a quality problem once at an arbitrary time.

We consider that one countermeasure resolves the quality problem. Of course, more than one countermeasure can be implemented in practice. The second countermeasure is considered after observing the effect of the first countermeasure. Therefore, only the first countermeasure is considered here.

4. The effect of a countermeasure appears by the next decision point.

5. The amount of products under warranty $n_{t}$ in the future is assumed by the planned schedule of production.

6. The decision-making model presented here adopts a descrete time model.

The time $t$ is between the present time $t_{0}$ and the time of the final production

$T$. Because there is no production after $T$ and a countermeasure afterward has no effect, we do not consider the pediod after $T$.

Under these assumptions, we consider whether to implement a countermeasure now or postpone the decision to the next decision point, and provide the decision support by comparing the quality cost of both cases quantitatively.

\section{Algorithm}

In this section, we illustrate our algorithm for solving the decision tree. In the following article, we use a discrete time domain. We calculate the expectation of loss amount caused by postponing a decision at the present time $t_{0}$. If the loss amount is greater than zero, implementing a countermeasure at $t_{0}$ is suggested.

\subsection{Flow chart of the presented algorithm}

Figure 3 illustrates the flow chart of the presented algorithm described in the next subsection. Our algorithm consists of five steps. As step 1, data and parameters are given as input: $r_{t}$ $\left(t \leq t_{0}\right), R_{t_{0}}, n_{t}\left(t \leq t_{0}\right), N_{t_{0}}, w, C, Q$, and $T$. Step 2 is setting the true claim rate $q_{i}^{*}$ by sampling from distribution $g\left(p^{*}\right)$ and generating future scenarios $\operatorname{Pr}\left(\hat{p}_{t+1} \mid \hat{p}_{t}, q_{i}^{*}\right)$. In step 3 , 


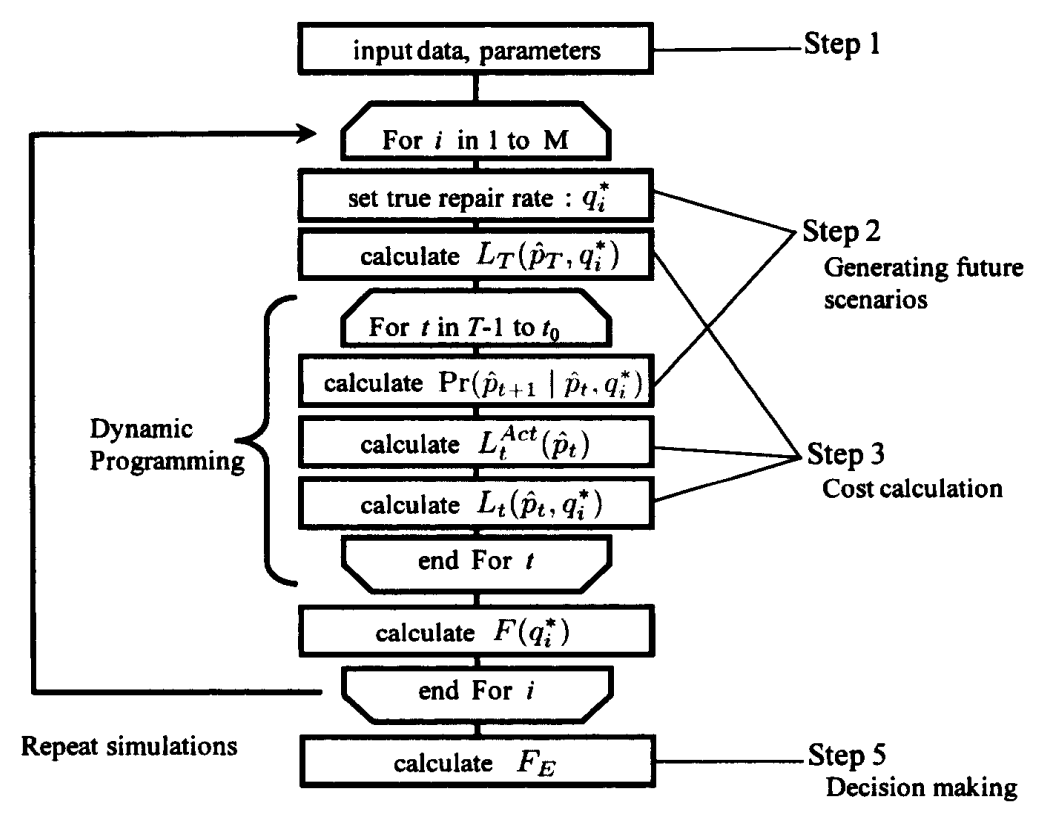

Figure 3: Flow chart of the presented algorithm

we calculate the quality cost under the future scenarios by dynamic programming. In step 4 , these steps are repeated $M$ times with different $q_{i}^{*}$. Step 5 calculates $F_{E}$ which is the expected loss amount caused by postponing the decision at $t_{0}$. Following these steps, the decision making is supported by the quantitative evaluation of quality cost considering the option to postpone.

\subsection{Detail of the proposed algorithm}

\section{Step 1. Input data and parameters}

As parameters, values of $w, C, Q$, and $T$ are given. The following data are given as input.

- $r_{t}\left(t \leq t_{0}\right), R_{t_{0}}$ : observed past amount of claims before the present time $t_{0}$.

- $n_{t}\left(t \leq t_{0}\right), N_{t_{0}}$ : observed past amount of in-warranty products before $t_{0}$.

\section{Step 2. Generating future scenarios}

In this step, we generate future claim scenarios based on the unobserved true claim rate $p^{*}$ and the future in-warranty amount $n_{t}\left(t>t_{0}\right)$ given by Assumption 5 . Here, we apply a Bayesian statistical approach. Because there is no clue to tell the true claim rate $p^{*}$ before any field claims are observed, we suppose that the prior distribution of claim rate is uniform distribution $\operatorname{Beta}(1,1)^{\dagger}$. The likelihood of the observed data $N_{t_{0}}, R_{t_{0}}$ with respect to $p^{*}$ is described with Binomial distribution $\operatorname{Bi}\left(N_{t_{0}}, p^{*}\right)^{\ddagger}$. Note that the observed claim rate $\hat{p}_{t_{0}}=R_{t_{0}} / N_{t_{0}}$ is only a maximum likelihood estimate of the claim rate. Then, the posterior distribution $g\left(p^{*}\right)$ is given by the following Beta distribution using Bayes' theorem ${ }^{\S}$.

$$
g\left(p^{*}\right) \sim \operatorname{Beta}\left(R_{t_{0}}+1, N_{t_{0}}-R_{t_{0}}+1\right) .
$$

Although the posterior distribution is given by Equation (3.1), the true claim rate is an unobservable fixed value. We set the true claim rate $p^{*}$ by sampling repeatedly at random from distribution $g\left(p^{*}\right)$, and we call its $i$ th value $q_{i}^{*}(i=1, \ldots, M)$. Then, the future scenario

${ }^{\dagger}$ The prior distribution can be any distribution if it is appropriate.

${ }^{\ddagger}$ The claim generation model at $t$ is considered to be given by the binomial distribution $\operatorname{Bi}\left(N_{t}, p^{*}\right)$.

${ }^{\S}$ This Bayesian idea is similar to [21]. 
is generated by calculating the following transition probability $\operatorname{Pr}\left(\hat{p}_{t+1} \mid \hat{p}_{t}, q_{i}^{*}\right)$. This is the conditional probability that the observed claim rate at $t+1$ is $\hat{p}_{t+1}=R_{t+1} / N_{t+1}$ when the observed claim rate at $t$ is $\hat{p}_{t}=R_{t} / N_{t}$. Here, $r_{t+1}=R_{t+1}-R_{t}=N_{t+1} \hat{p}_{t+1}-N_{t} \hat{p}_{t}$.

$$
\begin{aligned}
\operatorname{Pr}\left(\hat{p}_{t+1} \mid \hat{p}_{t}, q_{i}^{*}\right) & =\left(\begin{array}{c}
N_{t+1}-N_{t} \\
R_{t+1}-R_{t}
\end{array}\right) q_{i}^{*\left(R_{t+1}-R_{t}\right)}\left(1-q_{i}^{*}\right)^{\left(N_{t+1}-N_{t}\right)-\left(R_{t+1}-R_{t}\right)} \\
& =\left(\begin{array}{c}
n_{t+1} \\
r_{t+1}
\end{array}\right) q_{i}^{* r_{t+1}}\left(1-q_{i}^{*}\right)^{\left(n_{t+1}-r_{t+1}\right)}
\end{aligned}
$$

Following Assumption 1, the true claim rate is constant with respect to $t$. As mentioned in Section 2, the claim rate is segmented into discrete small grids between 0 and 1 . Therefore, $\hat{p}_{t}$ and $\hat{p}_{t+1}$ are discrete, and transition probability is calculated for finite scenario arcs.

Step 3. Cost calculation under the generated future scenario

In this step, we calculate the quality cost for each of the generated future scenarios. First, we calculate the expectation of quality cost of products after the present time $t_{0}$ when a countermeasure is implemented at $t$ and the observed claim rate is $\hat{p}_{t}$.

$$
\begin{aligned}
L_{t}^{A c t}\left(\hat{p}_{t}\right)=C & +w \int_{Q}^{1}\left(\left(n_{T}-n_{t}\right) Q+\left(n_{t}-n_{t_{0}}\right) p^{*}\right) \operatorname{Pr}\left(p^{*} \mid \hat{p}_{t}\right) d p^{*} \\
& +w \int_{0}^{Q}\left(n_{T}-n_{t_{0}}\right) p^{*} \operatorname{Pr}\left(p^{*} \mid \hat{p}_{t}\right) d p^{*} \\
=C & +w\left(\left(n_{T}-n_{t}\right) \delta_{t}\left(\hat{p}_{t}\right) Q+\left(n_{t}-n_{t_{0}}\right) \epsilon_{t}\left(\hat{p}_{t}\right)+\left(n_{T}-n_{t_{0}}\right)\left(\frac{R_{t}+1}{N_{t}+2}-\epsilon_{t}\left(\hat{p}_{t}\right)\right)\right) .
\end{aligned}
$$

Note that the true claim rate $p^{*}$ is unobservable. The first term in Equation (3.3) is the necessary cost of a countermeasure. The second term is the claim cost when the countermeasure is effective. The claim rate of products after the countermeasure becomes $Q$ (Assumption 2 ). The claim rate of products before the countermeasure does not change. The third term is the claim cost when the countermeasure is ineffective. The claim rate does not change and stays the same $p^{*}$ in this case. $\operatorname{Pr}\left(p^{*} \mid \hat{p}_{t}\right)$ is the conditional probability that the true claim rate is $p^{*}$ when the observed claim rate is $\hat{p}_{t}$. By Bayes' theorem,

$$
\begin{aligned}
\operatorname{Pr}\left(p^{*} \mid \hat{p}_{t}\right) & =\frac{\operatorname{Pr}\left(\hat{p}_{t} \mid p^{*}\right) g\left(p^{*}\right)}{\int_{0}^{1} \operatorname{Pr}\left(\hat{p}_{t} \mid p^{*}\right) g\left(p^{*}\right) d p^{*}} \\
& =\frac{\left(\begin{array}{l}
N_{t}-N_{t_{0}} \\
R_{t}-R_{t_{0}}
\end{array}\right) p^{*\left(R_{t}-R_{t_{0}}\right)}\left(1-p^{*}\right)^{\left(\left(N_{t}-N_{t_{0}}\right)-\left(R_{t}-R_{t_{0}}\right)\right)} g\left(p^{*}\right)}{\int_{0}^{1}\left(\begin{array}{l}
N_{t}-N_{t_{0}} \\
R_{t}-R_{t_{0}}
\end{array}\right) p^{*\left(R_{t}-R_{t_{0}}\right)}\left(1-p^{*}\right)^{\left(\left(N_{t}-N_{t_{0}}\right)-\left(R_{t}-R_{t_{0}}\right)\right)} g\left(p^{*}\right) d p^{*}} .
\end{aligned}
$$

$\delta_{t}\left(\hat{p}_{t}\right)$ in Equation (3.4) is the probability that the true claim rate $p^{*}$ is higher than the claim rate after countermeasure $Q: \delta_{t}\left(\hat{p}_{t}\right)=\operatorname{Pr}\left(p^{*}>Q \mid \hat{p}_{t}\right)$. When the observed claim rate is $\hat{p}_{t}$ and the countermeasure is implemented at $t$, we assume that the claim rate after the countermeasure will become $Q$ with probability $\delta_{t}\left(\hat{p}_{t}\right)$ calculated as follows (Assumption 2).

$$
\begin{aligned}
\delta_{t}\left(\hat{p}_{t}\right) & \equiv \int_{Q}^{1} \operatorname{Pr}\left(p^{*} \mid \hat{p}_{t}\right) d p^{*} \\
& =\frac{1}{\mathrm{~B}\left(R_{t}+1, N_{t}-R_{t}+1\right)} \int_{Q}^{1} p^{* R_{t}}\left(1-p^{*}\right)^{\left(N_{t}-R_{t}\right)} d p^{*}
\end{aligned}
$$


This is the upper probability of Beta distribution $\operatorname{Beta}\left(R_{t}+1, N_{t}-R_{t}+1\right)$. The probability density function of Beta distribution $\operatorname{Beta}(\alpha, \beta)$ is $f(x ; \alpha, \beta)=\frac{1}{\mathrm{~B}(\alpha, \beta)} x^{\alpha-1}(1-x)^{\beta-1}$. Beta function $\mathrm{B}(x, y)$ is $\mathrm{B}(x, y)=\int_{0}^{1} t^{(x-1)}(1-t)^{(y-1)} d t$. $\delta_{t}\left(\hat{p}_{t}\right)$ reflects the uncertainty of the claim rate. Even if $\hat{p}_{t}$ is high, $\delta_{t}\left(\hat{p}_{t}\right)$ is small at the beginning of the production period owing to the small amount of observed claims and in-warranty products. $\epsilon_{t}\left(\hat{p}_{t}\right)$ is defined as below and also calculated by the upper probability of Beta distribution.

$$
\begin{aligned}
\epsilon_{t}\left(\hat{p}_{t}\right) & \equiv \int_{Q}^{1} p^{*} \operatorname{Pr}\left(p^{*} \mid \hat{p}_{t}\right) d p^{*} \\
& =\frac{R_{t}+1}{N_{t}+2} \frac{1}{\mathrm{~B}\left(R_{t}+2, N_{t}-R_{t}+1\right)} \int_{Q}^{1} p^{*\left(R_{t}+1\right)}\left(1-p^{*}\right)^{\left(N_{t}-R_{t}\right)} d p^{*}
\end{aligned}
$$

$L_{t}\left(\hat{p}_{t}, q_{i}^{*}\right)$ is the quality cost based on the decision at $t$ when the observed claim rate is $\hat{p}_{t}$ and the claim scenario follows the unobservable true claim rate $q_{i}^{*}$. At each decision point, "the quality cost after $t_{0}$ in the case of implementing a countermeasure at $t$ " and "the quality cost after $t_{0}$ in the case of postponing the decision to $t+1$ " are compared. The optimum policy is to select the option with the smaller cost. When the decision is postponed, different $\hat{p}_{t+1}$ is observed at $t+1$ by the future scenarios (Equation (3.2)). Therefore, based on the decision at $t$, the quality cost $L_{t}\left(\hat{p}_{t}, q_{i}^{*}\right)$ is the smaller of $L_{t}^{A c t}\left(\hat{p}_{t}\right)$ and the expectation of $L_{t+1}\left(\hat{p}_{t+1}, q_{i}^{*}\right)$ about $\hat{p}_{t+1}$. In practice, the latter is evaluated discretely.

$$
L_{t}\left(\hat{p}_{t}, q_{i}^{*}\right)=\min \left(L_{t}^{A c t}\left(\hat{p}_{t}\right), \int_{0}^{1} \operatorname{Pr}\left(\hat{p}_{t+1} \mid \hat{p}_{t}, q_{i}^{*}\right) L_{t+1}\left(\hat{p}_{t+1}, q_{i}^{*}\right) d \hat{p}_{t+1}\right) .
$$

As shown in the above equation, in order to calculate $L_{t}\left(\hat{p}_{t}, q_{i}^{*}\right)$, we must calculate $L_{t+1}\left(\hat{p}_{t+1}, q_{i}^{*}\right)$ beforehand. Because there is no production after $T$ and a countermeasure afterward has no effect, no countermeasure will be implemented after $T$. Therefore?, $L_{T}\left(\hat{p}_{T}, q_{i}^{*}\right)$ can be calculated first based on the observation $\hat{p}_{T}$ by Bayes' theorem (Equation (3.5).

$$
L_{T}\left(\hat{p}_{T}, q_{i}^{*}\right)=w\left(n_{T}-n_{t_{0}}\right) \frac{R_{T}+1}{N_{T}+2}
$$

Here, $\frac{R_{T}+1}{N_{T}+2}$ is the mean of $\operatorname{Beta}\left(R_{T}+1, N_{T}-R_{T}+1\right)$. Consequently, tracking back from $T-1$ to $t_{0}$, calculation of $L_{t}\left(\hat{p}_{t}, q_{i}^{*}\right)$ is possible. This is realized by dynamic programming.

A decision at $t_{0}$ is made by comparing "the cost of implementing a countermeasure at $t_{0}$ " and "the cost of postponing the decision to $t_{0}+1$ ". We select the policy with the smaller cost. $F\left(q_{i}^{*}\right)$ below is the gap of the cost of both cases when the true claim rate is $q_{i}^{*}$. This is the loss amount caused by postponing the decision to the next decision point $t_{0}+1$. If $F\left(q_{i}^{*}\right)$ is positive, a countermeasure should be implemented at $t_{0}$. Otherwise, the decision should be postponed to $t_{0}+1$. This is also evaluated discretely in practice.

$$
F\left(q_{i}^{*}\right)=\int_{0}^{1} \operatorname{Pr}\left(\hat{p}_{t_{0}+1} \mid \hat{p}_{t_{0}}, q_{i}^{*}\right) L_{t_{0}+1}\left(\hat{p}_{t_{0}+1}, q_{i}^{*}\right) d \hat{p}_{t_{0}+1}-L_{t_{0}}^{A c t}\left(\hat{p}_{t_{0}}\right) .
$$

\section{Step 4. Repeat the simulations and cost calculations}

The discussion above is based on the situation that the true claim rate sampled randomly from the distribution $g\left(p^{*}\right)$ is $q_{i}^{*}$. We repeat Step 2 and Step $3 M$ times ( $M$ is a large enough number) and calculate $F\left(q_{i}^{*}\right)$ with respect to each $q_{i}^{*}(i=1, \ldots, M)$.

\footnotetext{
${ }^{\pi} L_{T}$ does not depend on the scenarios gererated by $q_{i}^{*}$. We represent it as $L_{T}\left(\hat{p}_{T}, q_{i}^{*}\right)$ for the sake of convenience.
} 


\section{Step 5. Decision making at present time $t_{0}$}

Finally we calculate the expectation $F_{E}$ of cost gap $F\left(q_{i}^{*}\right) . F_{E}$ means the expected loss amount caused by implementing no countermeasure at $t_{0}$ and postponing the decision to $t_{0}+1$. If $F_{E}$ is positive, implementing a countermeasure at $t_{0}$ is the optimum policy. If $F_{E}$ is negative, postponing the decision to $t_{0}+1$ is the optimum policy.

$$
F_{E}=\int_{0}^{1} F\left(p^{*}\right) g\left(p^{*}\right) d p^{*} \sim \frac{1}{M} \sum_{i=1}^{M} F\left(q_{i}^{*}\right)
$$

The quality cost at $t_{0}$ is given by Equation (3.8).

$$
L_{t_{0}}\left(\hat{p}_{t_{0}}, q_{i}^{*}\right)=\min \left(L_{t_{0}}^{A c t}\left(\hat{p}_{t_{0}}\right), \int_{0}^{1} \operatorname{Pr}\left(\hat{p}_{t_{0}+1} \mid \hat{p}_{t_{0}}, q_{i}^{*}\right) L_{t_{0}+1}\left(\hat{p}_{t_{0}+1}, q_{i}^{*}\right) d \hat{p}_{t_{0}+1}\right)
$$

This is also calculated with respect to each $q_{i}^{*}$. Therefore, the expected cost at $t_{0}$ is

$$
L_{t_{0} E}=\int_{0}^{1} L_{t_{0}}\left(\hat{p}_{t_{0}}, p^{*}\right) g\left(p^{*}\right) d p^{*} \sim \frac{1}{\mathrm{M}} \sum_{i=1}^{\mathrm{M}} L_{t_{0}}\left(\hat{p}_{t_{0}}, q_{i}^{*}\right)
$$

\section{Experiment}

\subsection{Decision making for different in-warranty numbers}

In this subsection, we show an experimental result of our presented method about the relationship between the number of in-warranty products and the decision-making results.

When there are many product models in the market, it is helpful to show the priority of the countermeasure for each of the product models. Manufacturers must implement efficient countermeasures for the product models with quality problems. In practice, in view of resource constraints, it may be impossible to implement countermeasures for all of the field problems.

As an experiment, we consider the situation that there are the four product models listed in Table 2 in the market. The observed claim rate and the production plan of these four product models are the same. The only difference of these product models is the inwarranty amount at the present time. The monthly production plans are evenly split by the simulation time step. Other parameters used commonly in all the experiments in this section are as follows. The simulation time step is two days, $t_{0}=0, T=45, w=20,000$, $Q=600 \mathrm{ppm}, N_{t_{0}}=n_{t_{0}} \times 15$. The number of grids that segment the claim rate is $k=654$, which is also the number of scenarios. The number of simulation is $M=654$.

Figure 4 illustrates the relationship of the cost of implementing a countermeasure $(C)$ and the loss amount caused by postponing the decision $\left(F_{E}\right)$. If $F_{E}$ is positive, implementing a countermeasure at $t_{0}$ is better than postponing the decision to $t_{0}+1$. If $F_{E}$ of a machine is positive when $C$ is very large, a countermeasure should be implemented for the product model even at a high cost. Therefore, we set the priority order by the value of $C$ when $F_{E}=0$. Figure 4 shows that the priority order is the same as the descending order of the in-warranty amount $n_{t}$ : D-C-B-A. This is because the situation is less uncertain if the in-warranty amount is large. The option to postpone is selected if there is high uncertainty.

If the cost $C$ of each product model is known, it is possible to determine the priority order by directly comparing $F_{E}$ at every known $C$. However, $C$ is usually unknown. Thus, it is effective to determine the priority order based on the value of $C$ when $F_{E}=0$. 
Table 2: Input data of the evaluated product models with different in-warranty amounts

\begin{tabular}{|c|c|c|c|c|c|}
\hline Product model & In-warranty & Observed claim & \multicolumn{2}{|c|}{ Production plan (in 1-3 months later) } \\
\cline { 4 - 6 } name & amount $n_{t_{0}}$ & rate $[\mathrm{ppm}] \hat{p}_{t_{0}}$ & 1 & 2 & 3 \\
\hline $\mathrm{A}$ & 3,000 & 800 & 3,000 & 4,000 & 5,000 \\
\hline $\mathrm{B}$ & 4,000 & 800 & 3,000 & 4,000 & 5,000 \\
\hline $\mathrm{C}$ & 8,000 & 800 & 3,000 & 4,000 & 5,000 \\
\hline $\mathrm{D}$ & 100,000 & 800 & 3,000 & 4,000 & 5,000 \\
\hline
\end{tabular}

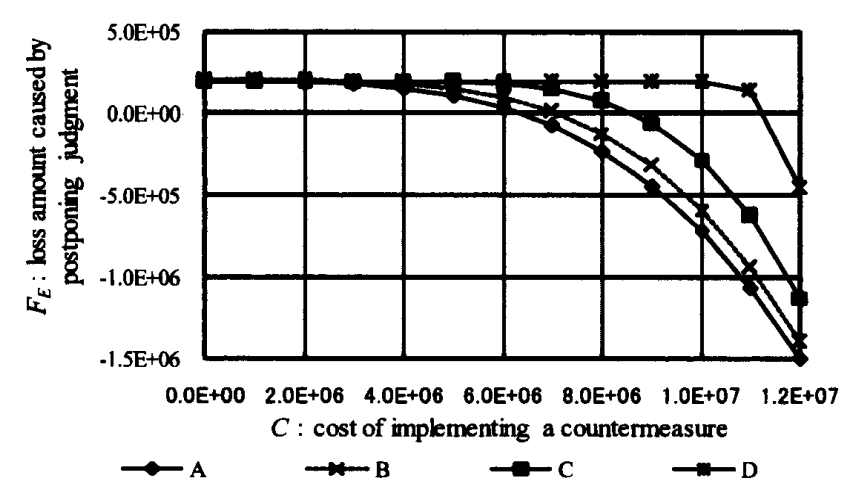

Figure 4: Cost of a countermeasure and loss of postponement (in-warranty amounts)

The calculation time for the production model $\mathrm{A}$ on a computer running Windows $\mathrm{XP}^{\mathrm{TM}}$ and MATLAB ${ }^{\mathrm{TM}}$ with an Intel Core $25^{\mathrm{TM}} 2.40 \mathrm{GHz}$ processor and $2.85 \mathrm{~GB}$ RAM was 88.2 seconds. If we set $M=69$ and $k=69$, the calculation time is 1.2 seconds and the difference of calcuated $L_{t_{0} E}$ is less than $1 \%$ for every $C$. Increasing $M$ and $k$ increases the accuracy of calcuated $L_{t_{0} E}$ slightly while the calculation time increases enormously.

\subsection{Value evaluation of the option to postpone}

In this subsection, we evaluate the value of the option to postpone. By using the data listed in Table 2 in the previous subsection, we compare the cost of the three cases below:

- With the option to postpone: $L_{t_{0} E}$. There is an opportunity to implement a countermeasure at the future decision point.

- Without the option to postpone: $L_{t_{0} E}^{N o O p t}$. A countermeasure can be implemented only at the present time $t_{0}$.

- Ideal judgment: $L_{t_{0} E}^{I d e a l}$. True claim rate $q_{i}^{*}$ is observable and decision is made by directly comparing $Q$ and $q_{i}^{*}$ at the present time $t_{0}$. This is an unrealistic situation.

$L_{t_{0} E}$ is calculated by Equation (3.13). $L_{t_{0} E}^{N o O p t}$ is calculated as follows. If there is no option to postpone and no countermeasure is implemented at all, the expected cost is calculated as follows based on the observed claim rate.

$$
L_{t_{0}}^{N o A c t}\left(q_{i}^{*}\right)=w\left(n_{T}-n_{t_{0}}\right) \frac{R_{t_{0}}+1}{N_{t_{0}}+2} .
$$

If a countermeasure is implemented at $t_{0}$, the cost is (See Equation (3.4))

$$
L_{t_{0}}^{A c t}\left(\hat{p}_{t_{0}}\right)=C+w\left(\left(n_{T}-n_{t_{0}}\right) \delta_{t_{0}}\left(\hat{p}_{t_{0}}\right) Q+\left(n_{T}-n_{t_{0}}\right)\left(\frac{R_{t_{0}}+1}{N_{t_{0}}+2}-\epsilon_{t_{0}}\left(\hat{p}_{t_{0}}\right)\right)\right) .
$$

We compare $L_{t_{0}}^{N o A c t}\left(q_{i}^{*}\right)$ and $L_{t_{0}}^{A c t}\left(\hat{p}_{t_{0}}\right)$.

$$
L_{t_{0}}^{N o O p t}\left(\hat{p}_{t_{0}}, q_{i}^{*}\right)=\min \left(L_{t_{0}}^{\text {NoAct }}\left(q_{i}^{*}\right), L_{t_{0}}^{A c t}\left(\hat{p}_{t_{0}}\right)\right) .
$$


Model A

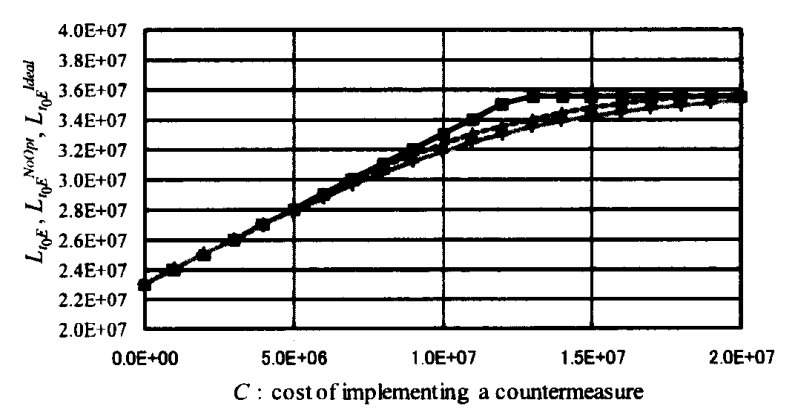

Model C

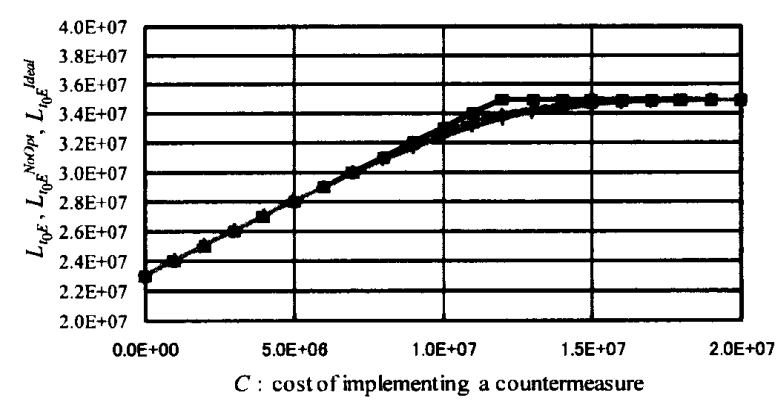

Model B

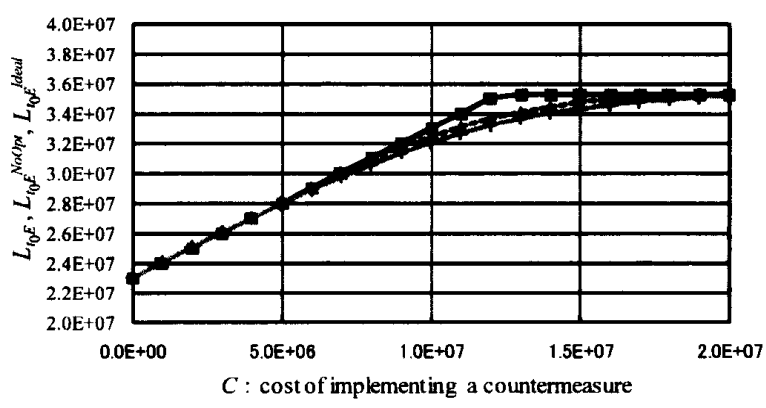

Model D

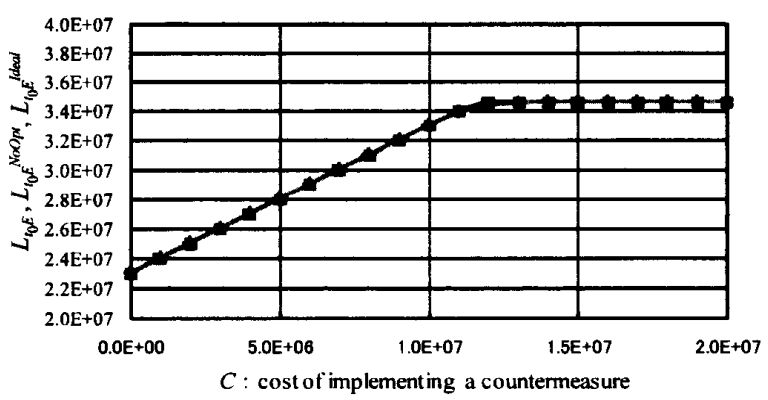

Figure 5: $L_{t_{0} E}$ (dotted lines), $L_{t_{0} E}^{N o O p t}$ (solid lines) and $L_{t_{0} E}^{I d e a l}$ (gray lines)

This depends on $q_{i}^{*}$ and the expectation is calculated as follows.

$$
L_{t_{0} E}^{N o O p t}=\int_{0}^{1} L_{t_{0}}^{N o O p t}\left(\hat{p}_{t_{0}}, p^{*}\right) g\left(p^{*}\right) d p^{*} \sim \frac{1}{M} \sum_{i=1}^{M} L_{t_{0}}^{N o O p t}\left(\hat{p}_{t_{0}}, q_{i}^{*}\right) .
$$

If $q_{i}^{*}$ is observable, decision can be made by directly comparing $Q$ and $q_{i}^{*}$.

$$
L_{t_{0}}^{\text {Ideal }}\left(\hat{p}_{t_{0}}, q_{i}^{*}\right)=\min \left(w\left(n_{T}-n_{t_{0}}\right) Q, w\left(n_{T}-n_{t_{0}}\right) q_{i}^{*}\right) .
$$

This depends on $q_{i}^{*}$ and the expectation is calculated as follows.

$$
L_{t_{0} E}^{\text {Ideal }}=\int_{0}^{1} L_{t_{0}}^{\text {Ideal }}\left(\hat{p}_{t_{0}}, p^{*}\right) g\left(p^{*}\right) d p^{*} \sim \frac{1}{M} \sum_{i=1}^{M} L_{t_{0}}^{\text {Ideal }}\left(\hat{p}_{t_{0}}, q_{i}^{*}\right) .
$$

Now, we show the value of the option to postpone experimentally. Again, we consider the situation that the four product models listed in Table 2 are in the market. Figure 5 shows the quality $\operatorname{cost}\left(L_{t_{0} E}, L_{t_{0} E}^{N o O p t}, L_{t_{0} E}^{\text {Ideal }}\right)$ with respect to the cost of implementing a countermeasure $(C)$. Dotted lines mean $L_{t_{0} E}$, solid lines mean $L_{t_{0} E}^{\text {NoOpt }}$ and gray lines mean $L_{t_{0} E}^{I d e a l}$. These graphs show that $L_{t_{0} E}$ is always smaller than $L_{t_{0} E}^{N o O p t}$ and larger than $L_{t_{0} E}^{I d e a l}$ for all $C$.

$$
L_{t_{0} E}^{N o O p t} \geq L_{t_{0} E} \geq L_{t_{0} E}^{\text {Ideal }}
$$

This result means that the consideration of the uncertainty is effective and the option to postpone has the possibility of reducing the quality cost.

The difference between $L_{t_{0} E}^{N o O p t}$ and $L_{t_{0} E}$ is smaller if there are more in-warranty products. This difference illustrates the value of the option to postpone. Figure 5 shows that the option to postpone is more valuable when the uncertainty at the present time $t_{0}$ is larger. 
Table 3: Input data of the evaluated product models with different observed claim rates

\begin{tabular}{|c|c|c|c|c|c|}
\hline Product model & In-warranty & Observed claim & \multicolumn{3}{|c|}{ Production plan (in 1-3 months later) } \\
\cline { 4 - 6 } name & amount $n_{t_{0}}$ & rate $[\mathrm{ppm}] \hat{p}_{t_{0}}$ & 1 & 2 & 3 \\
\hline $\mathrm{A}$ & 3,000 & 800 & 3,000 & 4,000 & 5,000 \\
\hline $\mathrm{E}$ & 3,000 & 900 & 3,000 & 4,000 & 5,000 \\
\hline $\mathrm{F}$ & 3,000 & 1,000 & 3,000 & 4,000 & 5,000 \\
\hline
\end{tabular}

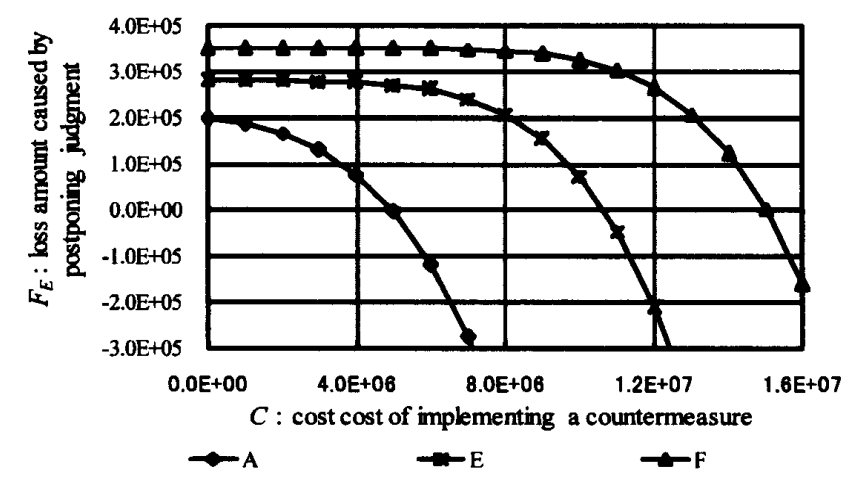

Figure 6: Cost of a countermeasure and loss of postponement (observed claim rates)

The difference between $L_{t_{0} E}^{N o O p t}$ and $L_{t_{0} E}$ is negligible when $C$ is large or small. This is because the decisions are the same with or without the option to postpone. When $C$ is small, a countermeasure is implemented immediately with or without the option to postpone. When $C$ is large, no countermeasure is implemented at all. When $C$ is a medium value, the option to postpone is valuable and a gap exists between $L_{t_{0} E}^{N o O p t}$ and $L_{t_{0} E}$.

The difference between $L_{t_{0} E}^{\text {Ideal }}$ and $L_{t_{0} E}$ is also smaller if there are more in-warranty products. This means that the decision with the option to postpone becomes more accurate when the uncertainty at the present time $t_{0}$ is smaller.

\subsection{Decision making for different observed claim rates}

In this subsection, we show another experimental result. Here, we examine the relationship between the observed claim rates and the decision-making results. As an experiment, we consider the situation that there are the three product models listed in Table 3 in the market. The number of in-warranty products and the production plan of these three product models is the same. The only difference of these product models is the observed claim rate at the present time. Other parameters are the same as in the previous subsection 4.1.

Figure 6 illustrates the relationship between the cost of a countermeasure for quality problem $(C)$ and the loss amount caused by postponing the decision $\left(F_{E}\right)$. If $F_{E}$ is positive even when $C$ is very large, a countermeasure should be implemented at any cost. Therefore, we set the priority order by the value of $C$ when $F_{E}=0$. Figure 6 shows that the priority order is high when the observed claim rate is high.

If the observed claim rate is high, the value along the vertical axis $\left(F_{E}\right)$ is also high as shown in Figure 6. In this experimental case, we can also decide the priority order by comparing $F_{E}$ directly. However, as shown in the previous subsection 4.1 (Figure 4), it is better to decide the priority order by the value of $C$ when $F_{E}=0$. 
Table 4: Input data of the evaluated product models with different production schedules

\begin{tabular}{|c|c|c|c|c|c|}
\hline \multirow{2}{*}{$\begin{array}{c}\text { Product model } \\
\text { name }\end{array}$} & In-warranty & Observed claim & \multicolumn{2}{|c|}{ Production plan (in 1-3 months later) } \\
\cline { 4 - 6 } & amount $n_{t_{0}}$ & rate $[\mathrm{ppm}] \hat{p}_{t_{0}}$ & 1 & 2 & 3 \\
\hline $\mathrm{A}$ & 3,000 & 800 & 3,000 & 4,000 & 5,000 \\
\hline $\mathrm{G}$ & 3,000 & 800 & 4,000 & 3,000 & 5,000 \\
\hline $\mathrm{H}$ & 3,000 & 800 & 5,000 & 3,000 & 4,000 \\
\hline $\mathrm{I}$ & 3,000 & 800 & 3,600 & 4,800 & 6,000 \\
\hline $\mathrm{J}$ & 3,000 & 800 & 4,200 & 5,600 & 7,000 \\
\hline
\end{tabular}
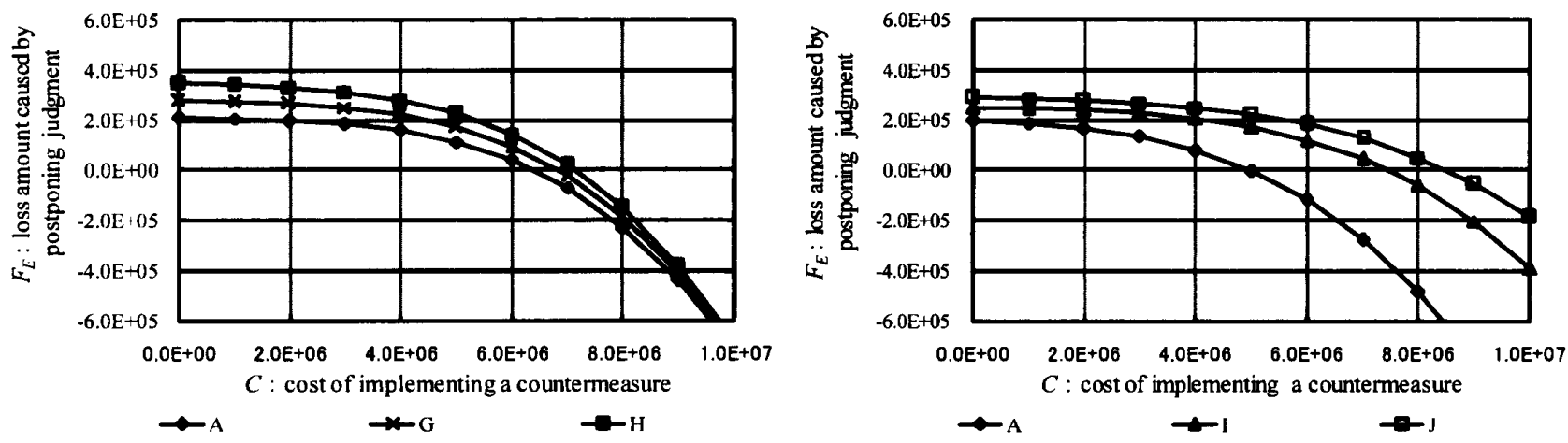

Figure 7: Cost of a countermeasure and loss of postponement (production plans)

\subsection{Decision making for different production plans}

In this subsection, we examine the relationship between the production plans and the decision-making results.

First, we consider the situation that there are the three product models A, G, H listed in Table 4 in the market. The only difference of these product models is the production schedules. The total production amounts are the same among these product models. Other parameters not listed below are the same as in the previous subsection 4.1. Figure 7 (left) shows that the priority order is high when the production amount in the next month is large. This is because the loss amount caused by postponing judgment depends on the production amount during the postponed decision time step. If the immediate future production increases, the loss amount caused by postponing judgment to the next decision point also increases.

Next, we consider the case when the total production amounts are not the same. We consider the situation that there are the three product models $\mathrm{A}, \mathrm{I}$, J listed in Table 4 in the market. Other parameters are the same as previously described in subsection 4.1. Figure 7 (right) shows that the priority order is high when the future production amount is large.

\subsection{Decision making in practice}

In practice, there are various product models in the market. Figure 8 is the case that the three product models listed in Table 5 exist in the market. Various lines are drawn in Figure 8 . The priority order of countermeasure is D-E-H based on the value of $C$ when $F_{E}=0$.

\section{Conclusion}

We proposed a method of decision support under uncertainty by considering the option to postpone the decision. In practice, the proposed method is useful for making a prompt 
Table 5: Input data of the evaluated product models with different production plans

\begin{tabular}{|c|c|c|c|c|c|}
\hline Product model & In-warranty & Observed claim & \multicolumn{3}{|c|}{ Production plan (in 1-3 months later) } \\
\cline { 4 - 6 } name & amount $n_{t_{0}}$ & rate $[\mathrm{ppm}] \hat{p}_{t_{0}}$ & 1 & 2 & 3 \\
\hline $\mathrm{D}$ & 100,000 & 800 & 3,000 & 4,000 & 5,000 \\
\hline $\mathrm{E}$ & 3,000 & 900 & 3,000 & 4,000 & 5,000 \\
\hline $\mathrm{H}$ & 3,000 & 800 & 5,000 & 3,000 & 4,000 \\
\hline
\end{tabular}

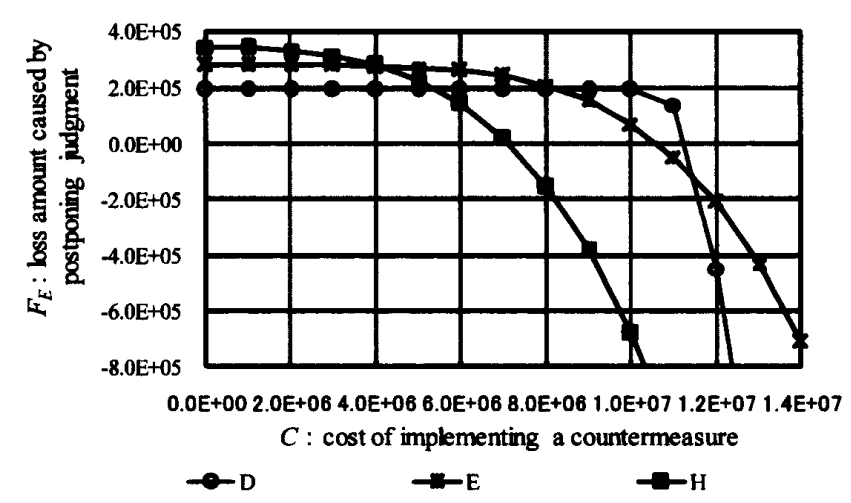

Figure 8: Cost of a countermeasure and loss of postponement

decision on implementing countermeasures for quality problems based on our filed quality data and shipment data, especially when the observed filed quality data is limited and uncertainty is large. The proposed method supports the prompt decision on whether to implement a countermeasure now or postpone the decision to the next decision point based on the scenario generated from observed data. We evaluated the option to postpone qualitatively and showed the possibility of reducing the quality cost. We also showed that the presented method is effective for making the priority order of product models in the market. Our goal is further practical application of the presented method. One of our future task is evaluating risk and unexpected loss, not the expectation of cost. The improvement of the assumption in the method is also our future work.

\section{References}

[1] M. Armstrong, A. Galli, W. Bailey and B. Couët: Incorporating technical uncertainty in real option valuation of oil projects. Journal of Petroleum Science and Engineering, 44-1-2 (2004), 67-82.

[2] R.E. Barlow and F. Proschan: Mathematical Theory of Reliability (John Wiley \& Sons, 1965).

[3] F. Black and M. Scholes: The pricing of options and corporate liabilities. Journal of Political Economy, 81-3 (1973), 637-654.

[4] M.J. Brennan and E.S. Schwartz: Evaluating natural resource investments. The Journal of Business, 58-2 (The University of Chicago Press, 1985), 135-157.

[5] Y.S. Chow, H. Robbins and D. Siegmund: Great Expectations: The Theory of Optimal Stopping (Houghton Mifflin, Boston, 1971).

[6] J.P. Decamps, T. Mariotti and S. Villeneuve: Investment timing under incomplete information. Mathematics of Operations Research, 30-2 (2005), 472-500.

[7] M.H. DeGroot: Optimal Statistical Decisions (McGraw Hill, New York, 1970). 
[8] A.K. Dixit, and R.S. Pindyck: Investment under Uncertainty (Princeton University Press, 1994).

[9] K. Handa, S. Matsumoto, M. Nakamoto, and N. Uchihira: An optimization method for investment and maintenance planning of power plants under uncertain environments. IEICE Transactions on Fundamentals of Electronics, Communications and Computer Sciences, E88-A-6 (2005), 1481-1486.

[10] S.D. Jacka: Optimal stopping and the American put. Mathematical Finance, 1 (1991), $1-14$.

[11] I. Karatzas: On the pricing of American options. Applied Mathematics 8 Optimization, 17 (1988), 37-60.

[12] M. Klein: Comment on "Investment timing under incomplete information". Mathematics of Operations Research, 34-1 (2009), 249-254.

[13] R.L. McDonald and D.R. Siegel: Investment and the valuation of firms when there is an option to shut down. International Economic Review, 26 (1985), 331-349.

[14] M. W. Lund: Real options in offshore oil field development projects. Proceedings of the 3rd International Conference on Real Options, 6-8 June, Wassenaar, Holland (1999).

[15] R. Merton: The theory of rational option pricing. Bell Journal of Economic and Management Science, 4-1 (1973), 141-183.

[16] J. Mun: Real Option Analysis: Tools and Techniques for Valuing Strategic Investment and Decisions (John Wiley \& Sons, 2002).

[17] Y. Otsubo: Risk minimization in optimal stopping problem and applications. Journal of the Operations Research Society of Japan, 46-3 (2003), 342-352.

[18] J.L. Paddock, D.R. Siegel, and J.L. Smith: Option valuation of claims on real asset: The case of offshore petroleum leases. Quarterly Journal of Economics, 103 (1988), 479-508.

[19] R.S. Pindyck: Irreversibilities and the timing of environmental policy. Resource and Energy Economics, 22 (2000), 233-259.

[20] P.A. Samuelson: Rational theory of warrant pricing. Industrial Management Review, 6 (1965), 41-50.

[21] R.E. Schafer and N.D. Singpurwalla: A sequential Bayes procedure for reliability demonstration. Naval Research Logistics Quarterly, 17-1 (1970), 55-67.

[22] A. Suzuki and K. Sawaki: The pricing of callable perpetual American options. Transactions of the Operations Research Society of Japan, 49 (2006), 19-31.

[23] Warranty Week. Warranty claims \& accruals in financial statements. (2011). http://www.warrantyweek.com/

[24] D.J. White: Mean, variance and probabilistic criteria in finite Markov decision processes: a review. Journal of Optimization Theory and Applications, 56 (1988) 1-29.

Minoru Nakatsugawa

Corporate Research \& Development Center,

Toshiba Corporation

1, Komukai Toshiba-cho, Saiwai-ku, Kawasaki-shi, Kanagawa, 212-8582, Japan

E-mail: minoru.nakatsugawa@toshiba.co.jp 